\title{
La salud pública en Jerez de los Caballeros (Badajoz) durante la Guerra de la Independencia
}

\author{
Suárez-Guzmán FJ. ${ }^{1}$, Peral Pacheco D. ${ }^{2}$
}

Sanid. mil. 2015; 71 (1): 60-65; ISSN: 1887-8571

\begin{abstract}
RESUMEN
La Guerra de la Independencia es un tema que se ha abordado en multitud de ocasiones. El objeto del presente trabajo es el estudio de la Salud Pública en Jerez de los Caballeros durante la Guerra de la Independencia española. Para ello hemos analizado los documentos del Archivo Histórico Municipal (AHMJC) y del Archivo Parroquial (APJC) de la ciudad. A pesar de hallar pocos fallecidos directamente a manos de las tropas francesas, las consecuencias en todos los ámbitos sobre la vida de la población fueron inmensas. Los ejércitos acampados en las cercanías de Jerez, aliados o no, demandaron enormes cantidades de alimentos y pertrechos a los jerezanos, los cuales ya estaban sumidos en la miseria. La destrucción de parte del AHMJC a manos de las tropas napoleónicas, conllevó la pérdida irremplazable de valiosos documentos.
\end{abstract}

PALABRAS CLAVE: Guerra de la Independencia, Salud Pública, Jerez de los Caballeros, Historia de la Medicina.

\section{Public health in Jerez de los Caballeros (Badajoz) during the War of Independence}

SUMMARY: The war of independence is an issue that has been addressed on numerous occasions. The object of this work is the study of the public health in Jerez de los Caballeros during the Spanish war of independence. To do this we have analyzed the documents of the Municipal historical archive (AHMJC) and of the parochial archive (APJC) city. Despite finding few deceased directly at the hands of the French troops, the consequences at all levels on the life of the people were huge. The armies camped in the outskirts of Jerez, allies or not, demanded huge amounts of food and ammunition to the Jerez, which were already mired in misery. The destruction of part of the AHMJC at the hands of Napoleon's troops, led to the irreplaceable loss of valuable documents.

KEYWORDS: War of independence. Public health. Jerez de los Caballeros. The History of Medicine.

\section{INTRODUCCIÓN}

En lo que respecta a la historiografía, se puede afirmar que ningún periodo de la Historia contemporánea española ha conocido la publicación de tantas obras como el de la Guerra de la Independencia $^{(1),(2),(3),(4),(5) .}$

(1) Sarmiento J. La Junta Suprema de Extremadura en la Guerra de la Independencia Española. Comisión de Gracia y Justicia (1802-1812). Badajoz. 2008. p. 24

(2) Blanco JP. Demografía, Familia y Sociedad en la Extremadura Moderna (1500-1860). Salamanca. 1999. p. 183.

(3) Sánchez J. Valladolid durante la Guerra de la Independencia Española (1808-1814). Biblioteca Virtual Cervantes. 2002.

(4) Anes G. Las Crisis Agrarias en la España Moderna. Madrid. 1970. p. 422 .

(5) González G. Jerez de los Caballeros en la Guerra de la Independencia. Tecnigraf, S. A. Madrid. 2008.

${ }^{1}$ Profesor Colaborador. Universidad de Extremadura. Médico de Familia. Centro Penitenciario de Badajoz. España.

${ }^{2}$ Profesor Titular. Universidad de Extremadura. Dpto. de Historia de la Ciencia. Badajoz. España.

Dirección para correspondencia: Fco. Javier Suárez-Guzmán. fcojsuarez@telefonica.net

Recibido: 7 de enero de 2014

Aceptado: 1 de abril de 2014
El objeto de nuestro estudio es Jerez de los Caballeros, ciudad situada al suroeste de la provincia de Badajoz, «...su clima es cálido, con vientos variables, aunque son mas permanentes el S., E. y O., y se padecen inflamatorias, intermitentes de todos tipos y carbunclos...» ${ }^{(6)}$. Figura 1.

La distribución de las calles y barrios sin un orden concreto «...revelan que su construcción fue siempre tan circunstanciosa como la necesidad del ensanche en cada momento histórico» ${ }^{(7)}$. Figura 2.

\section{OBJETIVOS}

Estudiar las repercusiones para la Salud Pública en Jerez de los Caballeros (Badajoz) durante la Guerra de la Independencia. Valorar las consecuencias del conflicto sobre sus habitantes.

\section{MATERIAL Y MÉTODO}

El estudio del AHMJC, y de los Libros de Difuntos de las cuatro parroquias jerezanas, ubicados en el APJC, así como publicaciones actuales y de la época.

(6) Madoz P. Diccionario Geográfico-Estadístico-Histórico de España y sus Posesiones de Ultramar. Tomo IX. Madrid. 1849. p. 626.

(7) Martínez-Martínez MR. El Libro de Jerez de los Caballeros. Enrique Rasco. Sevilla. 1892. Reeditado en 1993 por la Junta de Extremadura. p. 231. 


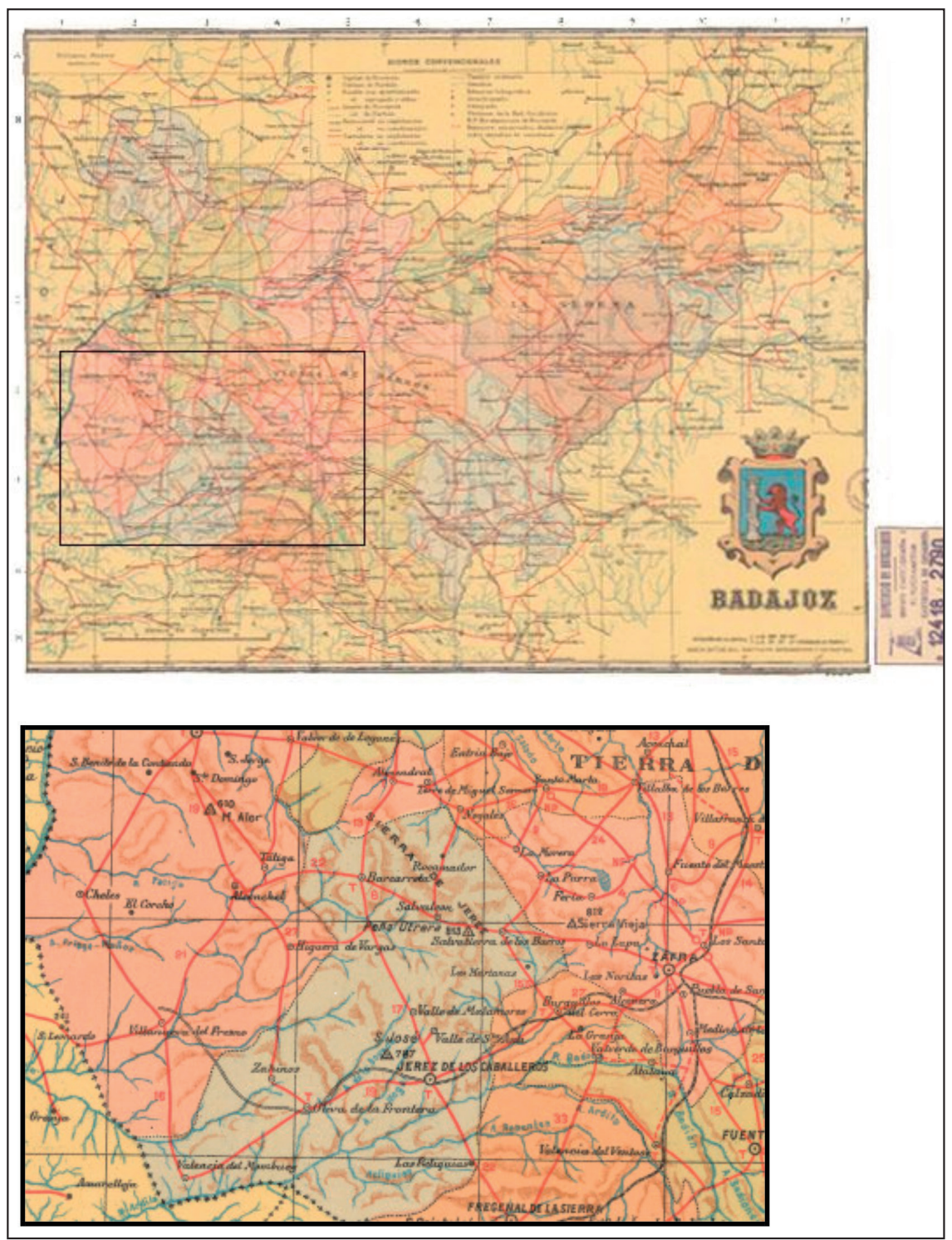

Figura 1. Situación geográfica de Jerez de los Caballeros en la provincia de Badajoz. Colección de Cartas Geográficas. Provincia de Badajoz. Instituto Geográfico y Estadístico. Barcelona. 1918.

\section{RESULTADOS}

Estalla la Guerra en mayo del 1808, perdurando hasta el 1814, suponiendo una gran pérdida de vidas y enormes alteraciones en la vida, la salud y la higiene de los jerezanos. La guerra contra los franceses va a sacudir poderosamente la vida en Jerez en todas sus facetas, y aunque el número de fallecidos directamente por la acción de los invasores sea escaso, los sufrimientos y pérdida de vidas indirectas fueron incontables, ya no solamente por las tropas napoleónicas, sino por las españolas e inglesas ${ }^{(8)}$.

El tratado de Fontainebleau en el 1807 permitió a los franceses atravesar España para invadir el Reino de Portugal, lo que facilitaría la ocupación de nuestro país por las tropas napoleónicas. Esto junto a las noticias procedentes de Bayona y el motín

(8) González G. Jerez de los Caballeros en la Guerra de la Independencia. Tecnigraf, S. A. Madrid. 2008. p. 12 de Aranjuez, desencadenan los sucesos del 2 de mayo en Madrid( ${ }^{(9)}$. La proclama del alcalde de Móstoles, que llamaba al levantamiento contra los enemigos, que se cree se supo en Badajoz por un carretero de Mérida, provoca la creación el día 6 de la Junta de Defensa, compuesta por personal del Ayuntamiento, clero, nobleza, letrados y labradores. Las tropas españolas se acantonan en Burguillos, necesitando suministros de las poblaciones cercanas continuamente ${ }^{(10)}$.

La extrema generosidad de particulares, nobles, plebeyos y religiosos, que en los primeros momentos aportaron dinero, ropas, armas y caballos, decae con el paso del tiempo. La sublevación de las provincias americanas desde Méjico a Argentina en el 1810, no sólo corta el flujo de recursos, sino que obliga a enviar tropas allí. Se puede concretar que, desde ese año, el Ejército se desenvuelve en la miseria más absoluta. Es normal que los soldados y aun los oficiales carezcan de calzado y que la dieta se redujera a pan y poco más ${ }^{(11)}$.

Durante la Guerra de la Independencia, el pueblo se vio obligado a surtir a los ejércitos acantonados en él, o cerca, de víveres y demás enseres necesarios para su manutención ${ }^{(12)}$. Debido a su situación geográfica, situado en una encrucijada de caminos entre la Sierra Morena y el Valle del Guadiana, su cercanía a Portugal y la amplitud de su término, Jerez se convirtió en lugar de paso de tropas españolas, francesas y británicas, las cuales se nutrían de los paupérrimos bolsillos de los ciudadanos, encontrándose el pueblo en un estado deplorable debido a $«$... los horrorosos saqueos que ha padecido la ciudad, de los cuantiosos suministros que ha hecho y de la escasez de numerario» ${ }^{(13)}$.

Las exigencias militares sobrepasaban frecuentemente las posibilidades de los pueblos, de esta manera, el 27 de abril del 1810 una orden del Ayuntamiento hace saber que se deben acreditar las reses aprehendidas para el Hospital Militar y las tropas, así como la obligación de no abandonar el pueblo, por lo menos hasta la entrada de los franceses, para que no falten pertrechos.

(9) Toro B. Zafra: Contribución Militar y Consecuencias de la Guerra de la Independencia. Actas del Congreso Internacional Guerra de la Independencia en Extremadura. IX Jornadas de Historia en Llerena. Llerena. 2008. p. 333.

(10) González G. Jerez de los Caballeros en la Guerra de la Independencia. Tecnigraf, S. A. Madrid. 2008. p. 31.

(11) Zorzo FJ. Las Operaciones Militares en la Guerra de la Independencia. Actas del Congreso Internacional Guerra de la Independencia en Extremadura. IX Jornadas de Historia en Llerena. Llerena. 2008. p. 32.

(12) Toro B. Zafra: Contribución Militar y Consecuencias de la Guerra de la Independencia. Actas del Congreso Internacional Guerra de la Independencia en Extremadura. IX Jornadas de Historia en Llerena. Llerena. 2008. p. 336.

(13) AHMJC. Gobierno Local. Libros de Acuerdos de Plenos. Legajo 19. Carpeta 130. Fols. 2 y 2 v. 


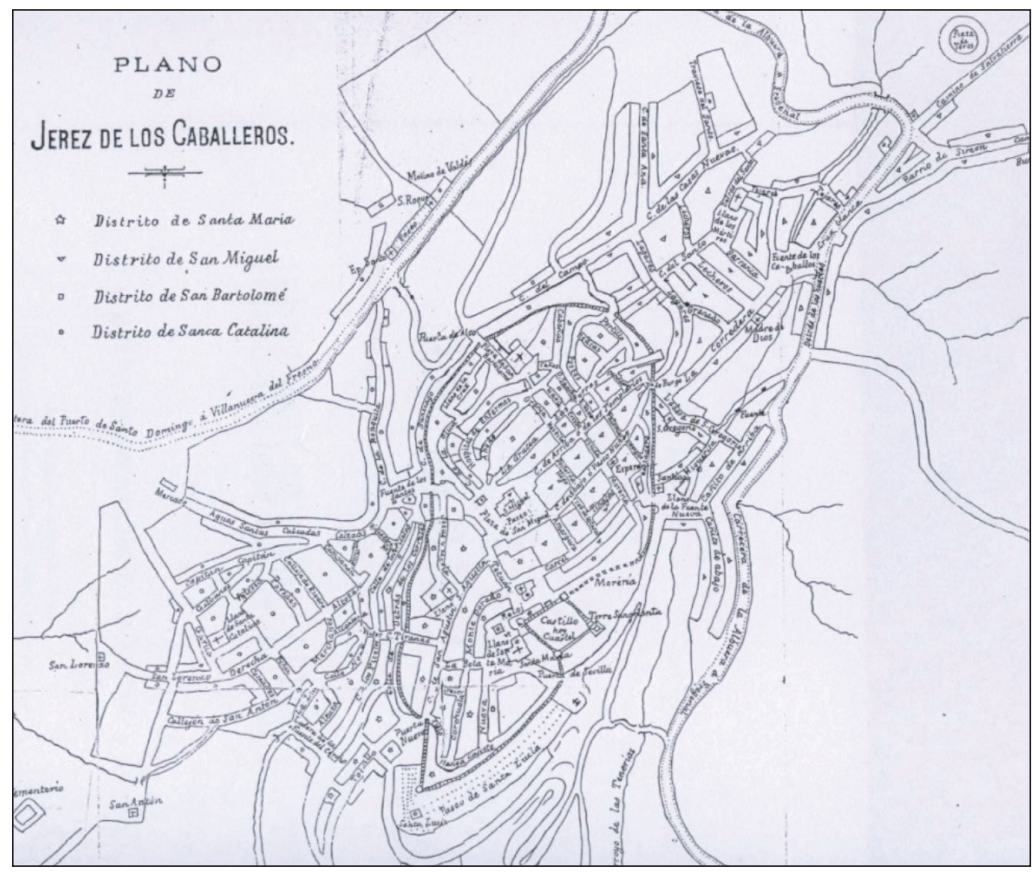

Figura 2. Plano de Jerez de los Caballeros en el 1892, en Martínez-Martínez MR. El Libro de Jerez de los Caballeros. Enrique Rasco. Sevilla. 1892. Reeditado en 1993 por la Junta de Extremadura. p. 515.

Además de nutrir a cualquier ejército que pasase por la ciudad, había que abastecer al Hospital Militar, instaurado recientemente ${ }^{(14)}$. En la ciudad previamente existían dos Hospitales: el de Enfermos y el de Transeúntes.

Los pedidos continuos y abusivos esquilman los escasísimos recursos de la población ${ }^{(15)}$, se suceden una y otra vez cada vez más exigentes y cargados de amenazas en caso de no cumplir$\mathrm{se}^{(16)}$. A pesar de las advertencias no siempre se podían cumplir las demandas, para reunir grano o ganado, que no hay o escasea, se necesita tiempo. Los ganaderos llegaron a dispersar sus reses por la multitud de dehesas del término, para que fuese más difícil localizarlas.

Se advierte que ciertos ayuntamientos como el del Valle de Matamoros, han incumplido la entrega de seis vacas para atender el suministro de las tropas, que están en Burguillos. Recordando a dichos alcaldes su responsabilidad, y como pueden ser conducidos presos a la cárcel si desobedecen ${ }^{(17)}$.

El 8 del mismo se le comunica al Mayor General del Ejército, sito en Burguillos «...la imposivilidad de poder subministrar (no con poco dolor de este Ayuntamiento) las raziones Trigo y tocino que pide diariamente para sobstener las Tropas de aque-

(14) Ibídem. Carpeta 128. Fol. 7.

(15) AHMJC. Órdenes. Organismos. Administración Provincial. Junta Suprema de la Provincia. Legajo 1. Carpeta 3. Sin foliar. El 16 de Diciembre del 1808 las Autoridades Provinciales mandan a pedir a todos los municipios de la provincia, que envíen todos los fusiles, garbanzos o trigo que puedan obtener, para las tropas que están en la zona de los puentes de Almaraz y del Arzobispo.

(16) González G. Jerez de los Caballeros en la Guerra de la Independencia. Tecnigraf, S. A. Madrid. 2008. p. 90.

(17) AHMJC. Gobierno Local. Libros de Acuerdos de Plenos. Legajo 19 Carpeta 128. Fol. 19. 4-V-1810. 1la División, por hallarse esta ciudad apurada de otras especies, a causa del crecido consumo de las Tropas... ${ }^{(18)}$. Así, prefieren abastecer el Hospital, que está con muchos enfermos, que a los soldados ante la carestía de alimentos. Ante esto la Junta de la Provincia socorre con algunos granos a la ciudad.

En la Tabla 1 podemos observar la petición de suministros exigidos por los ejércitos español y británico, aunque en esta ocasión no se puede discernir quién las efectuaba $^{(19)}$.

Ante tan desorbitadas exigencias, el Ayuntamiento no tiene más remedio que acudir a otros pueblos a comprar trigo y cebada. De esta manera, el 12 de abril del 1811 se envía un comisionado a la Parra, Salvatierra, Villalba de los Barros, Aceuchal y Almendralejo. Mediante la mediación del Conde de la Corte, vecino de Jerez, se consigue que Almendralejo permita sacar grano, lo cual estaba prohibido para evitar su escasez. En Villalba por cincuenta fanegas de trigo cobran cien reales por cada una; en Almendralejo por cien fanegas entre noventa y cuatro y noventa y cinco $^{(20)}$.

Se debe tener en cuenta que en el año del 1812 el precio del grano se llegó a multiplicar por cinco, debido a las crisis de subsistencias ${ }^{(21)}$.

El 4 de agosto del 1812, aparece la siguiente reseña, haciéndose notar «...los perjuicios que pueden originarse a la salud pública con la venta arbitraria de carne de todas especies que se está haciendo por falta de Abasto público sin presentar las reses de que procede para ser reconocidas, saber si son saludables, acreditar al mismo tiempo su legítima procedencia y arreglarles el precio del pormenor» ${ }^{(22)}$. Así, mediante edictos, se prohíbe que se pueda matar en las casas para vender al público: cabra, macho, carnero, ni oveja, debiendo llevarse las reses al matadero para ser reconocidas y averiguar su procedencia, y el precio al que se han comprado. Para ello acudirá a dicho matadero un Regidor o Diputado de Semana, e impondrá una multa de veinte ducados al que infrinja estas normas.

Como es lógico el Hospital tuvo mucho trabajo por esas fechas. Situado en la calle homónima, dentro del barrio de $\mathrm{S}$. Bartolomé, pero perteneciente a S. Miguel. Se trataba de un edificio de cortas dimensiones e inadecuado para sus funciones. Se ignora la fecha exacta de su fundación, creyéndose que ya existía en el siglo XV. Al fundarse el Convento de la Gracia existía el Hospital de S. Bartolomé, que quizás fuese el conocido en el siglo XIX como de Transeúntes; lo de denominarlo de S. Bartolomé a lo mejor se hizo para diferenciarlo del de S. Miguel, que podría existir en esa época. Siempre tuvo muchas rentas y mala administración $^{(23)}$.

(18) Ibídem. Fol. 22

(19) Extraído y modificado de González G. Jerez de los Caballeros en la Guerra de la Independencia. Tecnigraf S. A. Madrid. 2008. pp. 91-2.

(20) Ibídem. Fols. 128 y 128 v».

(21) Pérez V. Las Crisis de Mortalidad en la España Interior siglos XVI-XIX. Siglo XXI de España Editores. Madrid. 1980. p. 387.

(22) AHMJC. Gobierno Local. Libros de Acuerdos de Plenos. Legajo 19. Carpeta 132. Fol. 299.

(23) Martínez-Martínez MR. El Libro de Jerez de los Caballeros. Enrique Rasco. Sevilla. 1892. Reeditado en 1993 por la Junta de Extremadura. p. 329. 


\section{La salud pública en Jerez de los Caballeros (Badajoz) durante la Guerra de la Independencia}

El día 30 de abril del 1812 un auto dirigido al Barón del Solar de Espinosa, regente del Hospital de Enfermos, le informa de «...padeciendo enfermedades consecuentes de la agitación de las marchas y crueldad de las estaciones [...] hallandose enfermos un crecido número de soldados de su division, resistiendo sus males sobre una estera o tal vez en los humedos ladrillos, se sirba disponer que esta tarde a las 5 esten prontas sesenta y quatro camas completas para colocarlas en un edificio destinado ya a hospital de campaña... ${ }^{(24)}$. También se exige a los Alcaldes de Barrio que aporten: S. Miguel quince camas, Sta. Catalina once, Sta. María siete y S. Bartolomé otras siete; si es preciso se utilizará la fuerza para obtenerlas ${ }^{(25)}$.

Al no dársele el auxilio adecuado a los enfermos militares, como se había acordado, el 11 de junio del 1812 el cirujano al cargo dispone la marcha de éstos, no haciéndose responsable de las consecuencias. Este mismo día se le contesta desmintiendo tal cosa, y recriminándole las formas y el fondo de su auto, llamándole la atención sobre el mal uso de las instalaciones del Hospital por parte de los soldados enfermos ${ }^{(26)}$.

El 18 de agosto del 1812 aparece una nota del Comandante de las Armas de esta ciudad, haciendo ver que los enfermos del Hospital Militar Provisional, que se ha abierto de nuevo recientemente para atender a los soldados, disfrutan de dos raciones. También hace notar que el capellán del regimiento de caballería D. Sebastián Mulero subsiste de limosnas, estando asistiendo constantemente a los pacientes. Así, se sugiere que se supriman algunas raciones para dárselas al mencionado capellán ${ }^{(27)}$. El mismo día contesta con extrañeza el Ayuntamiento, ya que según la Constitución, le corresponde al comandante el Régimen Económico del Hospital Provisional, pudiendo dar y quitar las raciones que considere convenientes, tanto en el Hospital como fuera de él, sin que nadie de la Corporación pueda entorpecer$1 \mathrm{e}^{(28)}$.

Aunque no se ha carecido de médicos en la ciudad por haberlos proporcionado la fortuna, el Municipio ve arriesgado para los 1.600 habitantes (es a lo que ha quedado reducida la población a causa de la guerra), no tener una dotación económica segura para dos médicos y un cirujano latino ${ }^{(29)}$.

En cuanto a las causas de muerte hallamos cuatro fallecidos relacionados con la Guerra de la Independencia:

Muerte por el enemigo $1^{(30)}$

Muerte por los franceses $1^{(31)}$

Aporreada por los franceses $1^{(32)}$

Maltratado por invasión del enemigo $1^{(33)}$

(24) AHMJC. Gobierno Local. Libros de Acuerdos de Plenos. Legajo 19. Carpeta 132. Fols. 142 y 142 v».

(25) Ibídem. Fol. 143.

(26) Ibídem. Fols. 207-8.

(27) Ibídem. Fol. 311 v.

(28) Ibídem. Fol. 313.

(29) Ibídem. Legajo 20. Carpeta 134. Fols. 16-7.

(30) APJC. Parroquia de Sta. Catalina. Libro de Difuntos n 7 (1805-1833). 11-VII-1810.

(31) APJC. Parroquia de Sta. María. Libro de Difuntos nº 6 (1796-18315). 19-III-1812.

(32) APJC. Parroquia de Sta. Catalina. Libro de Difuntos n 7 (1805-1833). 6-VIII-1810.

(33) Ibídem. 20-III-1810.
Realmente los años de la contienda no presentan una mortalidad sobresaliente, en la Tabla 2 podemos contemplar la cantidad de fallecidos por año y las Tasas Brutas de Mortalidad (TBM), que es el $\mathrm{N}^{\circ}$ total de muertes en el año x 1000/ Población media de ese año.

Como se aprecia, exceptuando el año del 1812, los demás no presentan unas cifras muy llamativas. Debemos tener en cuenta que en Jerez, durante el siglo XIX, el año de mayor mortalidad fue el de 1894 con 466 óbitos y una TBM del 52,1\%o, y el de menor el del 1835 con 115 fallecidos y una TBM del 18,7\%o.

\section{DISCUSIÓN}

La base de nuestro trabajo es el estudio del AHMJC, parte del cual está destruido o perdido para siempre, y gran culpa de ello la tiene la ocupación napoleónica. Uno de los actos violentos más comunes realizados por las tropas francesas al acceder a una población, era el de asaltar y destruir todo o parte de los documentos custodiados en los archivos públicos y eclesiásticos. En el caso de Jerez de los Caballeros, tenemos noticias de estos actos en diversas fuentes. M. R. Martínez indica que: «Faltan los libros de Actas de la Junta desde Enero hasta Mayo de 1810, no hay testimonio directo que revele los desastres que sufrió Jerez en esos cuatro meses» ${ }^{(34)}$. El autor deduce que su ausencia se debe a que, al no encontrarse constituida la Junta de la ciudad por haber emigrado la mayoría de sus Autoridades, no existan libros por no ser posible la toma de acuerdos. No obstante, la Actas Municipales indican perfectamente en sus acuerdos del 11 de junio del 1811, los destrozos ocasionados por las tropas francesas en el archivo público calificándolo de esta manera: «... el estado de ruina en que los enemigos han quedado el Archivo Público [...] resulta imposibilidad de poder encontrar el libro correspondiente...» ${ }^{(35)}$.

La entrada de los franceses conlleva el saqueo, pillaje y destrucción, «...la ciudad de Jerez puede ufanarse de que participó sólo de sus desastres y amarguras, poniendo á servicio de la patria la sangre de sus vecinos y sufriendo con constancia los desmanes y las depredaciones del enemigo»(36).

La expulsión de los enemigos y liberación del país, supone el principio del fin de Napoleón:

«Esta maldita Guerra de España fue la causa primera de todas las desgracias de Francia. Todas las circunstancias de mis desastres se relacionan con este nudo fatal: destruyó mi autoridad moral en Europa, complicó mis dificultades, abrió una escuela a los soldados ingleses... esta maldita guerra me ha perdido» ${ }^{(37)}$.

La Nación está totalmente arruinada y la pérdida de las colonias americanas cercana, las cosechas y la ganadería destrozadas. Se ha ganado la guerra ¿pero a qué precio? El regreso del rey Fernando VII, el Deseado, supone la abolición de la Constitución

(34) Martínez-Martínez MR. El Libro de Jerez de los Caballeros. Enrique Rasco. Sevilla, 1892. Reeditado en 1993 por la Junta de Extremadura, p. 141. (35) AHMJC. Gobierno Local. Libros de Acuerdo de Plenos. Legajo 19. Carpeta 129. Fol. 20.

(36) Martínez-Martínez MR. El Libro de Jerez de los Caballeros. Enrique Rasco. Sevilla. 1892. Reeditado en 1993 por la Junta de Extremadura. p. 137. (37) Fraser R. La Maldita Guerra de España. Historia Social de la Guerra de la Independencia, 1808-1814. Prólogo. Editorial Verso. 2008. 
Tabla 1. Peticiones de Suministros Exigidos por el Ejército Español y Británico entre el 4 y el 15 de Abril del 1811.

\begin{tabular}{|c|c|c|}
\hline 4 de Abril & $\begin{array}{l}\text { Comisario de guerra del IV Ejército desde } \\
\text { Zafra }\end{array}$ & Medicinas diversas señaladas en una lista* \\
\hline 8 de Abril & General Ballesteros & $\begin{array}{l}1.000 \text { raciones de pan (cada tres días) } \\
1.000 \text { raciones de carne (cada tres días) }\end{array}$ \\
\hline 9 de Abril & $\begin{array}{l}\text { Luís del Corral, Comandante de los Húsares } \\
\text { de Castilla }\end{array}$ & $\begin{array}{l}50 \text { fanegas de cebada } \\
50 \text { ovejas o } 10 \text { vacas } \\
300 \text { raciones de pan }\end{array}$ \\
\hline 11 de Abril & $\begin{array}{l}\text { Comisario de Guerra del IV Ejército desde } \\
\text { Fregenal de la Sierra }\end{array}$ & $\begin{array}{l}300 \text { raciones de pan } \\
5 \text { fanegas de trigo o su equivalente en hierro } \\
50 \text { vacas }\end{array}$ \\
\hline 15 de Abril & Comisario de Guerra desde Zafra & $\begin{array}{l}500 \text { raciones de pan } \\
20 \text { vacas }\end{array}$ \\
\hline 16 de Abril & $\begin{array}{l}\text { Comisario de Guerra de la } 3^{\text {a }} \text { División del } \\
\text { IV Ejército desde Burguillos del Cerro }\end{array}$ & $\begin{array}{l}2.000 \text { raciones de pan } \\
600 \text { raciones de grano } \\
24 \text { vacas }\end{array}$ \\
\hline 17 de Abril & $\begin{array}{l}\text { Comisario de Guerra de la } 3^{\text {a }} \text { División del } \\
\text { IV Ejército desde Burguillos del Cerro }\end{array}$ & 8.000 raciones de pan de harina \\
\hline 17 de Abril & $\begin{array}{l}\text { Jefe del Estado Mayor del V Ejército del } \\
\text { General Castaños }\end{array}$ & $\begin{array}{l}1.000 \text { raciones diarias de pan } \\
400 \text { raciones diarias de forraje o grano }\end{array}$ \\
\hline 22 de Abril & $\begin{array}{l}\text { Comisario de Guerra de la } 3^{\text {a }} \text { División del } \\
\text { IV Ejército desde Fregenal de la Sierra }\end{array}$ & $\begin{array}{l}\text { Nuevamente la petición de las } 8.000 \text { raciones de pan del } \\
\text { día } 17\end{array}$ \\
\hline 22 de Abril & $\begin{array}{l}\text { Comisario General del Ejército Británico } \\
\text { desde el Cuartel General en Olivenza en } \\
\text { nombre del General Beresford }\end{array}$ & 400 cabezas de ganado diarias \\
\hline 25 de Abril & $\begin{array}{l}\text { Comisario del IV Ejército del General } \\
\text { Ballesteros desde Fregenal de la Sierra }\end{array}$ & $\begin{array}{l}100 \text { limones } \\
10 \text { libras de azúcar }\end{array}$ \\
\hline
\end{tabular}

Fuente: A.H.M.J.C.

* A.H.M.J.C. Gobierno Local. Libros de Acuerdos de Plenos. Legajo 19, Carpeta 130. Fol. 114. Algunas de las medicinas eran: palo dulce, malvavisco, halo santo rasurado, licor de azafrán, especies pectorales, alcanfor, ungüento de Arsorex, licor de Hosfam, alcalí volátil, vinagre, zarzaparrilla, corteza de canela entera, licor de manzanilla, tamarindo, mana, triaca, jabón medicinal, sal amoniaco, sal de nitro, manteca de puerco, miel, corteza de naranja, cabezas de adormidera, goma arábiga, mercurio crudo, agua de melisa espirituosa, flor de azufre, tierra foliada de tártaro, aceite común, etc.

Tabla 2. Mortalidad en Jerez de los Caballeros Durante la Guerra de la Independencia (1808-1814).

\begin{tabular}{lll}
\hline Años & Muertos & T. B. M. \%o \\
1808 & 199 & 27,6 \\
1809 & 290 & 40,5 \\
1810 & 292 & 41,0 \\
1811 & 213 & 30,1 \\
1812 & 371 & 52,7 \\
1813 & 207 & 29,6 \\
1814 & 229 & 32,9 \\
Total & 1.081 & 36,3 \\
\hline
\end{tabular}

Fuente: A. P. J. C. Libros de Difuntos. 1808-1814. 


\section{La salud pública en Jerez de los Caballeros (Badajoz) durante la Guerra de la Independencia}

promulgada por las Cortes de Cádiz en el 1812, y el retorno al Absolutismo anterior al 1808(38). Vuelve el Antiguo Régimen.

En las tres primeras décadas del siglo los porcentajes de fallecidos infantiles respecto al total de párvulos ${ }^{(39)}$, son abrumadores con cifras cercanas al 100\% (entre 1811 y 1820 alcanza el 96,4\%). Con tasas brutas de mortalidad que oscilan entre el 18,7\% del 1835 y el $52,7 \%$ del 1812 , es debido a las malas cosechas, las epidemias de hambre y miseria, y la Guerra de Independencia, con su cenit en el 1812 .

\section{CONCLUSIONES}

La Guerra de la Independencia supuso un auténtico desastre para Jerez de los Caballeros. Junto a las calamitosas circunstancias en las que se hallaba España, y por ende la población, asolada por sequías, epidemias y crisis de subsistencias, se añade la invasión de Napoleón. Los soldados franceses saquearon en varias ocasiones la ciudad, destruyendo parte del AHMJC, con la inherente pérdida irremplazable de documentos. Los diferentes ejércitos participantes en la contienda, exigían enormes cantidades de alimentos y suministros a Jerez y las poblaciones cercanas, para su abastecimiento, demandas que la mayoría de las veces no podían ser satisfechas y que cuando lo eran dejaban totalmente desprovistos de provisiones a sus habitantes. La mortalidad no fue mayor que otros periodos de la centuria, esto puede ser debido a un subregistro en las anotaciones parroquiales de los Libros de Difuntos, por diversas circunstancias.

(38) Fontana J. La Quiebra de la Monarquía Absoluta, 1814-1820. Editorial Ariel. Barcelona. 1974. pp. 79-90.

(39) Pérez V. La Crisis de Mortalidad en la España Interior siglos XVI-XIX. Siglo XXI de España Editores. Madrid. 1980. p. 36. Consideramos párvulo al periodo entre los 0 y 7 años de vida.

\section{BIBLIOGRAFÍA}

1. Sarmiento J. La Junta Suprema de Extremadura en la Guerra de la Independencia Española. Comisión de Gracia y Justicia (1802-1812). Badajoz. 2008.

2. Blanco JP. Demografía, Familia y Sociedad en la Extremadura Moderna (1500-1860). Salamanca. 1999.

3. Sánchez J. Valladolid durante la Guerra de la Independencia Española (18081814). Biblioteca Virtual Cervantes. 2002.

4. Anes G. Las Crisis Agrarias en la España Moderna. Madrid. 1970

5. González G. Jerez de los Caballeros en la Guerra de la Independencia. Tecnigraf, S. A. Madrid. 2008.

6. Madoz P. Diccionario Geográfico-Estadístico-Histórico de España y sus Posesiones de Ultramar. Tomo IX. Madrid. 1849.

7. Martínez-Martínez MR. El Libro de Jerez de los Caballeros. Enrique Rasco. Sevilla. 1892. Reeditado en 1993 por la Junta de Extremadura.

8. Instituto Geográfico y Estadístico. Barcelona. 1918.

9. Toro B. Zafra: Contribución Militar y Consecuencias de la Guerra de la Independencia. Actas del Congreso Internacional Guerra de la Independencia en Extremadura. IX Jornadas de Historia en Llerena. Llerena. 2008.

10. Zorzo FJ. Las Operaciones Militares en la Guerra de la Independencia. Actas del Congreso Internacional Guerra de la Independencia en Extremadura. IX Jornadas de Historia en Llerena. Llerena. 2008.

11. AHMJC. Gobierno Local. Libros de Acuerdos de Plenos.

12. AHMJC. Órdenes. Organismos. Administración Provincial. Junta Suprema de la Provincia.

13. Pérez V. Las Crisis de Mortalidad en la España Interior siglos XVI-XIX. Siglo XXI de España Editores. Madrid. 1980.

14. APJC. Libros de Difuntos.

15. Fraser R. La Maldita Guerra de España. Historia Social de la Guerra de la Independencia, 1808-1814. Prólogo. Editorial Verso. 2008.

16. Fontana J. La Quiebra de la Monarquía Absoluta, 1814-1820. Editorial Ariel. Barcelona. 1974. 